\title{
Tendances des visites aux services des urgences pour des intoxications liées à l'acétaminophène, 2011 à 2019
}

\author{
Jaskiran Kaur, M. Sc.; Steven R. McFaull, M. Sc.; Felix Bang, M. Santé publique
}

Diffuser cet article sur Twitter

\begin{abstract}
Résumé
Nous avons examiné les tendances dans les taux de consultation aux services des urgences pour une intoxication liée à l'acétaminophène au Canada. Au total, nous avons relevé 27123 cas d'intoxication dans les sites sentinelles de la base de données électronique du Système canadien hospitalier d'information et de recherche en prévention des traumatismes (eSCHIRPT) entre avril 2011 et février 2019, dont 13,7 \% étaient liés à l'utilisation d'acétaminophène. Nous avons observé une tendance décroissante significative des taux au sein de l'ensemble des intoxications involontaires (hommes : $-10,3 \%$; femmes : $-8,0 \%$ ). Pour les intoxications intentionnelles, une diminution significative a eu lieu chez les femmes $(-5,9 \%)$ uniquement. Les femmes ont toujours eu des taux plus élevés de consultation à un service des urgences pour des intoxications, tant involontaires qu'intentionnelles.
\end{abstract}

Mots-clés : acétaminophène, Tylenol, paracétamol, intoxication, SCHIRPT

\section{Introduction}

L'acétaminophène, aussi connu sous le nom de paracétamol, APAP $^{1}$ ou Tylenol, est un médicament couramment utilisé pour réduire la douleur et la fièvre. Il est facilement accessible sur le marché, dans de nombreux médicaments en vente libre et sur ordonnance. On le trouve sous forme de produit individuel contenant de l'acétaminophène seulement (p. ex. Tylenol), comme remède contre le rhume en vente libre (p. ex. DayQuil/NyQuil) ou en association avec un opiacé (p. ex. Percocet). En raison de sa grande disponibilité, l'acétaminophène est une cause courante d'ingestion, tant involontaire qu'intentionnelle.

Des recherches effectuées au Canada et aux États-Unis ont montré que, consommé en quantités excessives, l'acétaminophène est la principale cause d'insuffisance hépatique aiguë1-4. Environ 4500 hospitalisations dues à une surdose d'acétaminophène sont signalées chaque année au Canada.
Environ $6 \%$ des patients hospitalisés pour une surdose présentent des troubles hépatiques, en particulier une insuffisance hépatique aiguë pouvant entraîner la mort ${ }^{2}$.

Cet article vise à décrire le profil des cas d'ingestion d'acétaminophène signalés par les services des urgences canadiens.

\section{Méthodologie}

Le Système canadien hospitalier d'information et de recherche en prévention des traumatismes (SCHIRPT) ${ }^{5}$ est un système de surveillance sentinelle des blessures et des intoxications administré par l'Agence de la santé publique du Canada. Le SCHIRPT recueille des données auprès des services des urgences de 11 hôpitaux pédiatriques et de 8 hôpitaux généraux au Canada, soit 19 établissements au total. Nous avons effectué une recherche dans la base de données électronique (eSCHIRPT) pour obtenir les dossiers de visites à un service des urgences pour des intoxications

\section{Points saillants}

- Au total, nous avons relevé, sur les 27123 cas d'intoxication dans la base de données électronique du SCHIRPT, 3721 cas $(13,7 \%)$ liés à l'acétaminophène.

- Environ 50,3 \% des intoxications étaient involontaires, 48,6 \% intentionnelles et $1,1 \%$ d'intention indéterminée.

- Nous avons observé une tendance décroissante significative des taux d'intoxication à l'acétaminophène au sein des intoxications involontaires chez les hommes ( $-10,3 \%)$ comme chez les femmes $(-8,0 \%)$.

- Nous avons constaté une diminution significative des taux d'intoxication à l'acétaminophène au sein des intoxications intentionnelles chez les femmes $(-5,9 \%)$ mais pas chez les hommes.

- Comparativement aux hommes, les femmes présentaient des taux systématiquement plus élevés de consultation à un service des urgences pour des intoxications tant involontaires qu'intentionnelles liées à l'acétaminophène.

liées à l'acétaminophène entre le $1^{\mathrm{er}}$ avril 2011 et le 23 février 2019 ( $N=1037$ 843). Les cas ont été inclus si les critères suivants étaient respectés :

i. Description de la blessure

- La cause directe de la blessure, ou les facteurs contributifs, a été codée comme suit : " [Acétaminophène, 
INCL Tylenol - seul] » (code eSCHIRPT 753F) ou " [Acétaminophène, INCL Tylenol - avec d'autres substances, INCL Tylenol avec codéine] » (code eSCHIRPT 754F) ou " [Médicaments contre les allergies ou le rhume, INCL ceux contenant de l'AAS ou de l'acétaminophène] " (code eSCHIRPT 755F).

- Le texte narratif comprenait les mots clés ou les chaînes clés en français et en anglais qui suivent : « ACETA », « ACÉTA », « TYLENOL », " TYLÉN ", « PARACETA », « PARACÉTA ».

ii. Nature de la blessure et cause externe.

- La nature du code de blessure est notée comme intoxication ou effet toxique (code eSCHIRPT 50NI) ou la cause externe contient le code 301EC (301EC : [Intoxication INCL des drogues de rue et des médicaments sur ordonnance, de l'alcool, des solvants, des gaz, des substances corrosives et des pesticides ou des engrais]).

Dans les cas où un seul critère sur trois a été satisfait, un analyste a statué manuellement sur le cas. De plus, plusieurs cas ( $n=98$ ) ont été exclus de l'analyse car nous étions incertains du médicament consommé.

Nous avons analysé les tendances de 2011 à 2018 au moyen de la version 4.6.0.0 (SEERStat, NCI, Bethesda, Maryland, É.-U.) du Joinpoint Regression Program, qui produit la variation annuelle moyenne en pourcentage (VAMP) ou la variation annuelle en pourcentage (VAP) ${ }^{6}$. Les données pour 2019 sont incomplètes et ont donc été exclues de l'analyse des tendances. Toutes les analyses statistiques ont été effectuées à l'aide de la version 5.1 du logiciel Enterprise Guide (SAS Institute Inc., Cary, Caroline du Nord, États-Unis).

\section{Résultats}

Pour la période allant du $1^{\mathrm{er}}$ avril $2011 \mathrm{au}$ 23 février 2019, nous avons relevé dans les sites sentinelles de l'eSCHIRPT 27123 cas d'intoxication, dont 3721 (13,7\%) liés à l'acétaminophène, soit 13719 cas sur 100000 cas d'intoxication de l'eSCHIRPT. Environ 50,3 \% des intoxications ont été classées comme involontaires, 48,6 \% comme intentionnelles et $1,1 \%$ comme d'intention indéterminée.
L’âge médian était de 14,0 ans (intervalle interquartile [IIQ] : 2,0-16,0). Les femmes représentaient les deux tiers $(n=2513$; $67,5 \%$ ) des consultations à un service des urgences pour une intoxication liée à l'acétaminophène, soit 19,9\% de tous les cas d'intoxication chez les femmes pour 2001-2019. Les jeunes de 15 à 19 ans représentaient un tiers (35 \%) des cas d'intoxication liée à l'acétaminophène dans l'eSCHIRPT et les enfants de 2 à 4 ans, $22,9 \%$.

Les intoxications intentionnelles liées à l'acétaminophène étaient significativement plus élevées chez les 15 à 19 ans (28,2 \%; $p<0,0001)$ et chez les femmes $(42,2 \%$; $p<0,0001)$. La majorité des cas $(n=2597$; $69,8 \%$ ) concernaient des produits contenant de l'acétaminophène seulement (p. ex., Tylenol), 15,9\% $(n=590)$ des cas, de l'acétaminophène combiné à d'autres

FIGURE 1

Évolution des taux normalisés ${ }^{\mathrm{a}}$ de consultation à un service des urgences associée à une intoxication intentionnelle ou involontaire à l'acétaminophène, par modalité d'intention et par sexe, eSCHIRPT, 2001-2018

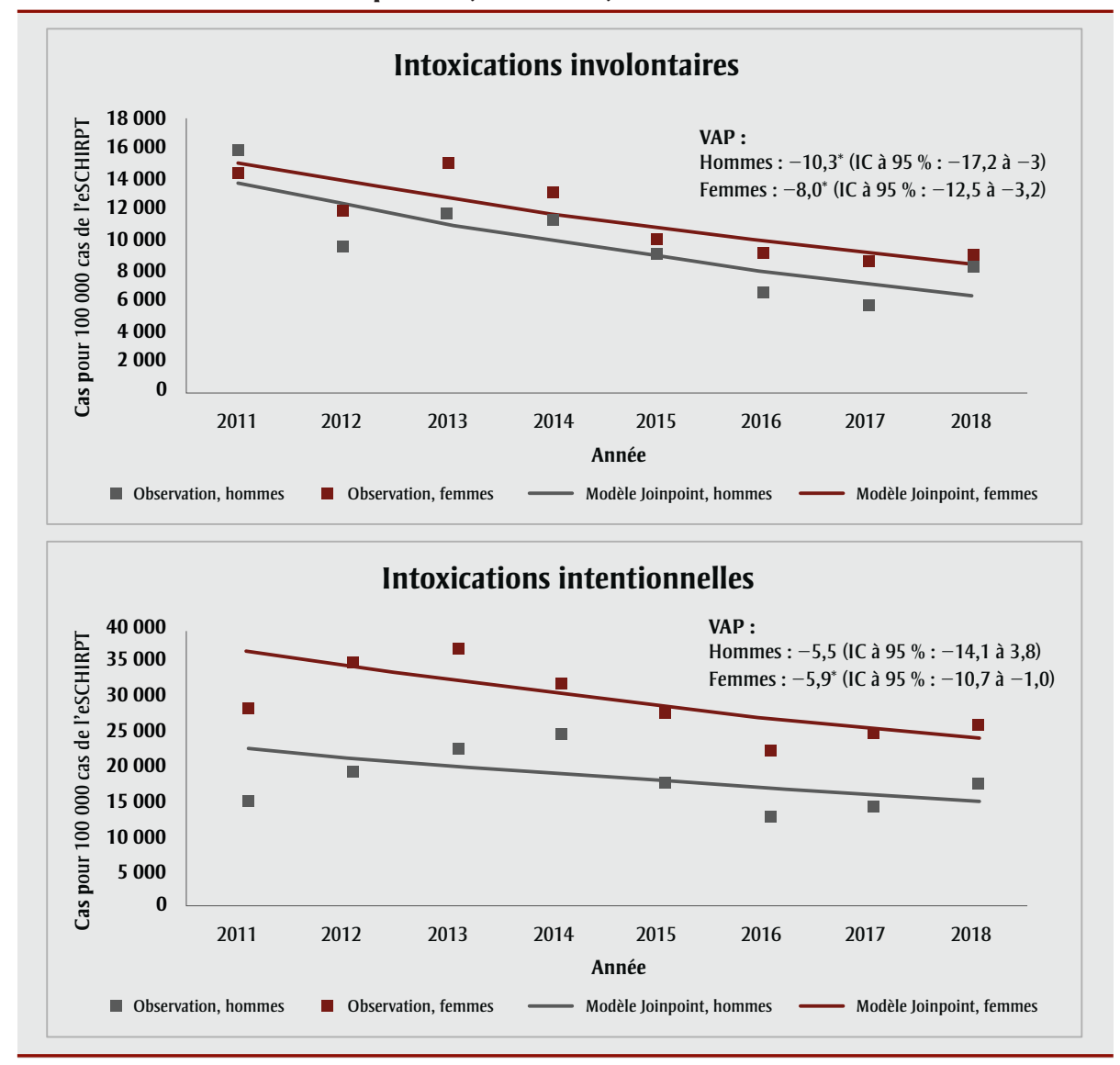

Abréviations : eSCHIRPT, Système canadien hospitalier d'information et de recherche en prévention des traumatismes électronique; IC, intervalle de confiance; VAP, variation annuelle en pourcentage.

Remarque : L'intention indéterminée a été exclue de l'analyse en raison du faible nombre de cas.

${ }^{a}$ Exprimée en tant que proportion de tous les cas de l'eSCHIRPT pour une année donnée ( $\left.\times 100000\right)$.

b Comme les données pour 2019 sont incomplètes, seules les données de 2011 à 2018 sont incluses dans l'analyse Joinpoint.

${ }^{*}$ La VAP diffère significativement de zéro au seuil $\alpha=0,05$. substances (p. ex., Tylenol avec de la codéine) et $14,4 \%(n=534)$, des médicaments contre les allergies, le rhume et la toux contenant de l'acétaminophène. Les intentionnelles liées à l'acétaminophène étaient discernables chez les La figure 1 montre les résultats de l'analyse au moyen du logiciel Joinpoint dintention et par sexe exclues de l'analyse des tendances). Nous trouvé aucun point d'inflexion et 列 involontaires, nous avons observé une tendance décroissante significative de la proportion d'intoxications liées à l'acétaminophène aussi bien chez les hommes ( $-10,3 \%$; intervalle de confiance sont incomplètes et par conséquent, 
[IC] à $95 \%$ : de $-17,2$ à $-3,0)$ que chez les femmes $(-8,0 \%$; IC à $95 \%$ : de $-12,5$ à $-3,2)$. Pour les intoxications intentionnelles à l'acétaminophène, une diminution significative a eu lieu chez les femmes $(-5,9 \%$; IC à $95 \%$ : de $-10,7$ à $-1,0)$, mais pas chez les hommes.

La figure 2 présente les résultats pour les deux sexes par tranche d'âge et par modalité d'intention. Les femmes sont plus âgées, avec un âge médian de 14,0 ans (IIQ : 9,0 à 16,0) par rapport à un âge médian de 3,0 ans pour les hommes (IIQ : 2,0 à 15,0). Les femmes ont toujours eu des taux plus élevés de consultation aux services des urgences que les hommes pour des intoxications à l'acétaminophène, aussi bien involontaires qu'intentionnelles. Avec un taux de près de six fois plus élevé que celui des garçons du même groupe d'âge, les filles de 10 à 19 ans ont un taux d'intoxication involontaire disproportionnellement élevé

FIGURE 2

Répartition normalisée ${ }^{\mathrm{a}}$ selon l'âge et le sexe des consultations à un service des urgences pour une intoxication liée à l'acétaminophène, eSCHIRPT, 2001-2019

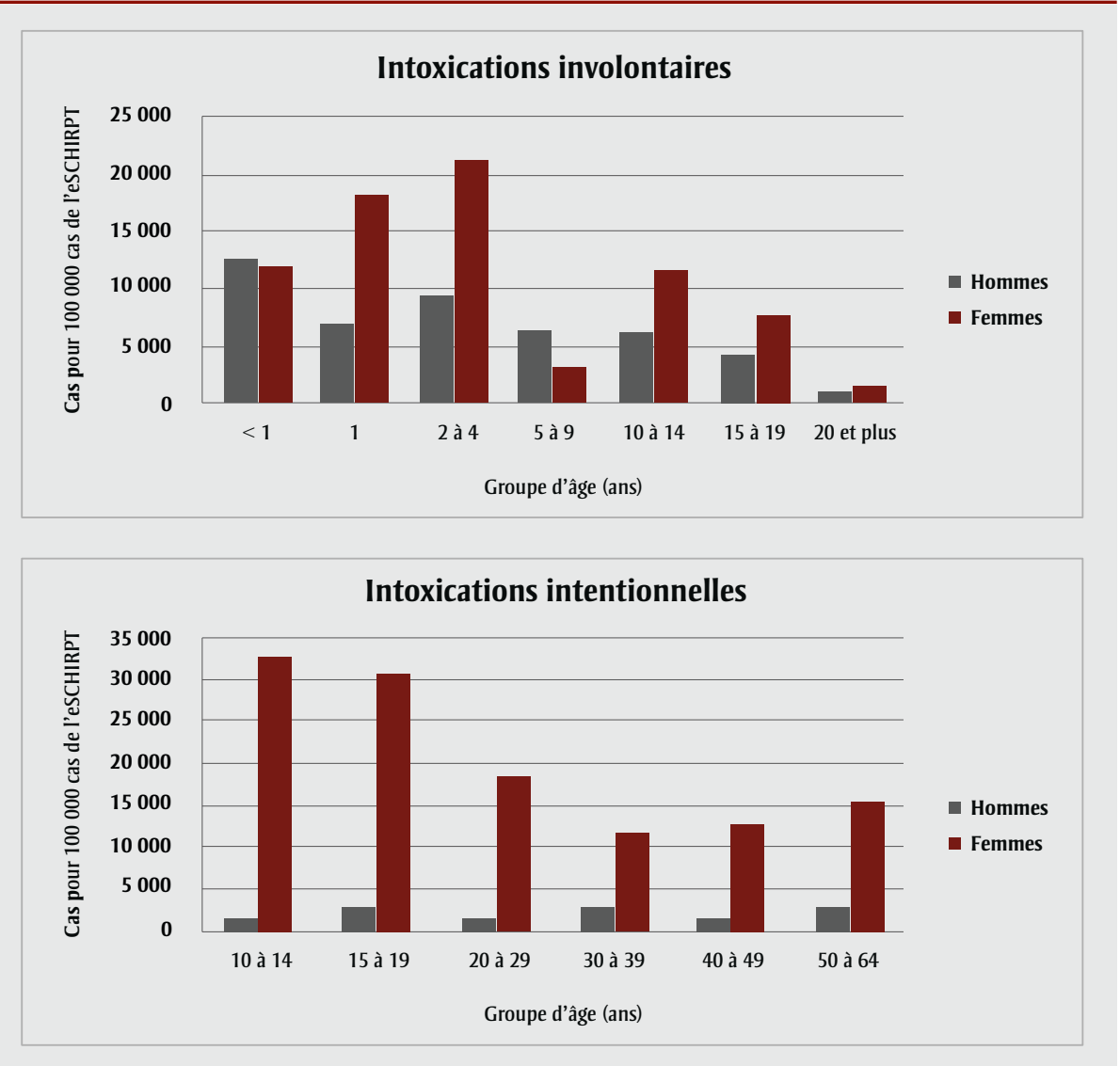

Abréviation : eSCHIRPT, Système canadien hospitalier d'information et de recherche en prévention des traumatismes électronique. Remarque : En raison de l'instabilité des données, les groupes d'âge des moins de 10 ans et des plus de 65 ans n'ont pas été inclus dans l'analyse sur l'intoxication intentionnelle.

${ }^{a}$ Exprimée en tant que proportion de tous les cas de l'eSCHIRPT dans une tranche d'âge donnée $(\times 100000)$. surdose d'acétaminophène semblent avoir diminué entre 2011 et 2018 . En proportion de l'ensemble des intoxications involontaires, on observe une tendance décroissante significative de la place de l'acétaminophène tant chez les hommes et les femmes alors que dans les cas d'intoxications intentionnelles, nous avons observé une diminution significative de la proportion liée à l'acétaminophène chez les femmes mais pas chez les hommes.

Nous avons constaté que les enfants de 0 à 4 ans avaient plus d'intoxications accidentelles liées à l'acétaminophène que les autres tranches d'âge. Les intoxications accidentelles prédominaient chez les filles et les femmes de tous les groupes d'âge, sauf chez les nouveau-nés et nourrissons de moins d'un an. Il est possible que ces surdoses accidentelles aient été causées par une erreur de calcul ou une mesure incorrecte des doses par les parents ou les tuteurs. Par contraste, l'intoxication intentionnelle par l'acétaminophène était plus commune chez les jeunes de 10 à 19 ans, avec une prévalence plus élevée chez les filles. Des études ont montré que l'autointoxication est la méthode la plus courante de tentative de suicide chez les adolescents $^{11,12}$ et que les médicaments en vente libre sont le moyen le plus couramment utilisé ${ }^{13}$. La mortalité liée à l'acétaminophène est ainsi plus élevée dans les pays où il est possible d'en obtenir des quantités illimitées ${ }^{14}$. Cependant, il est important de noter que l'utilisation de l'acétaminophène comme moyen d'autointoxication n'est pas seulement liée à son accessibilité, mais également à sa popularité et à la disponibilité d'autres méthodes.

En 2009, Santé Canada a finalisé la Norme d'étiquetage pour l'acétaminophène, ce qui a mené à l'inclusion d'avertissements plus sévères sur le risque de lésions au foie $^{15}$. En 2016, la norme d'étiquetage a été révisée en fonction des résultats de l'examen de 2014 de Santé Canada ${ }^{16}$. Les recommandations intègrent en particulier un avertissement plus sévère sur la consommation concomitante d'alcool, davantage d’information sur l'étiquette à propos de la sécurité du contenu et un tableau de données sur le médicament fournissant des directives et des avertissements dans un format facile à lire. Un dispositif de dosage a également été inclus pour aider les parents et les soignants à administrer les produits d'acétaminophène liquide pour enfants. 
Tous les produits à base d'acétaminophène doivent être conformes à la nouvelle norme d'étiquetage depuis mars 2018. Cette norme d'étiquetage améliorée a sans doute été mise en œuvre depuis trop peu de temps pour avoir un effet visible sur les données présentées ici. Il faut donc maintenir une surveillance continue des intoxications liées à l'acétaminophène pour évaluer l'effet de cette norme d'étiquetage améliorée et pour bonifier la mise en œuvre de mesures de sécurité visant à réduire le risque de lésion causée par ce médicament régulièrement utilisé et largement accessible.

\section{Points forts et limites}

Cette étude fournit l'analyse la plus récente des tendances pancanadiennes de consultation à un service des urgences pour une intoxication à l'acétaminophène. Cependant, notre échantillon n'est pas entièrement représentatif de la population canadienne car seuls certains hôpitaux participent au SCHIRPT. Or les sites du SCHIRPT sont principalement des hôpitaux pédiatriques situés dans de grandes villes et les adolescents plus âgés, les adultes, certains peuples autochtones et les habitants des régions rurales sont sous-représentés.

Même si l'eSCHIRPT consigne les cas de personnes décédées à leur arrivée aux urgences, la mortalité est sous-représentée puisque les données des services des urgences ne consignent ni les cas des personnes mortes avant d'avoir pu être transportées à l'hôpital ni les cas des personnes mortes après leur admission. Les patients qui sont admis aux urgences pour traitement immédiat sans passer par l'admission peuvent également ne pas être compilés. Il en va de même pour les personnes qui ne remplissent pas le formulaire de déclaration de blessure ou d'intoxication.

Enfin, les comptes rendus reposent en grande partie sur les déclarations des patients et des fournisseurs de soin, dans la mesure où ils sont uniquement composés des renseignements fournis au moment de la blessure. Les patients qui ne connaissent pas bien les médicaments peuvent confondre l'acétaminophène avec d'autres médicaments. De plus, l'intention d'intoxication peut aussi être potentiellement faussée par omission, les patients étant susceptibles ne pas vouloir la déclarer en raison de la nature délicate du sujet.

\section{Remerciements}

Les auteurs tiennent à remercier Melinda Tiv et James Cheesman pour leur précieuse contribution aux règles de codage et de classification des cas d'intoxication liée à l'acétaminophène. Ils souhaitent également remercier Aimée Campeau pour sa relecture du manuscrit.

\section{Conflits d'intérêts}

Aucun.

\section{Contributions des auteurs et avis}

JK a effectué l'analyse des données et a rédigé le manuscrit. $\mathrm{FB}$ a effectué l'analyse au moyen du logiciel Joinpoint. SRM et FB ont contribué à la relecture du manuscrit.

Tous les auteurs sont employés de l'Agence de la santé publique du Canada. Cependant, le contenu de l'article et les points de vue qui y sont exprimés n'engagent que les auteurs et ne correspondent pas nécessairement à ceux du gouvernement du Canada.

\section{Références}

1. Lee MW. Acetaminophen (APAP) hepatoxicity - Isn't it time for APAP to go away? J Hepatol. 2017;67(6): 1324-1331. doi:10.1016/j.jhep.2017.07 .005 .

2. Santé Canada. Acétaminophène [Internet]. Ottawa (Ont.) : Gouvernement du Canada; 2016 [consulté le 10 avril 2019]. En ligne à : https://www .canada.ca/fr/sante-canada/services /medicaments-et-appareils-medicaux /acetaminophene.html

3. Yoon E, Babar A, Choudhary M, Kutner M, Pyrsopoulos N. Acetaminophen-induced hepatotoxicity: a comprehensive update. J Clin Transl Hepatol. 2016;4(2):131-42. doi:10.14218 /JCTH.2015.00052.

4. Clark R, Fisher JE, Sketis IS, Johnston GM. Population prevalence of high dose paracetamol in dispensed paracetamol/opiod prescription combinations: an observational study. BMC Clin Pharmacol. 2012;12(11). doi:10.1186 /1472-6904-12-11.
5. Crain J, McFaull S, Thompson W, et al. Le Système canadien hospitalier d'information et de recherche en prévention des traumatismes : un système de surveillance des blessures dynamique et novateur. Promotion de la santé et prévention des maladies chroniques au Canada. 2016;36(6): 127-133. doi:10.24095/hpcdp.36.6.02f.

6. Statistical Methodology and Applications Branch, Surveillance Research Program, National Cancer Institute, Joinpoint regression program, version 4.6.0.0 - avril 2018. Bethesda (MD) : National Cancer Institute; 2018.

7. Myers RP, Li B, Shaheen AA, et al. Emergency department visits for acetaminophen overdose: a Canadian population-based epidemiologic study (1997-2002). CJEM. 2007;9(4):267-274.

8. Major MJ, Zhou HE, Wong HL, et al. Trends in rates of acetaminophen-related adverse events in the United States, Pharmacoepidemiol Druf Saf. 2016;25(5):590-598. doi:10.1002/pds .3906 .

9. Skinner R, McFaull S, Rhodes AE, Bowes M, Rockett IR. Suicide in Canada: is poisoning misclassification an issue? Can J Psychiat. 2016; 61(7):405-412. doi:10.1177/07067437 16639918 .

10. Skinner R, McFaull S, Draca J, et al. Le suicide et les hospitalisations associées à des blessures auto-infligées au Canada (1979 à 2014-2015). Promotion de la santé et prévention des maladies chroniques au Canada. 2016;36(11):272-281.

11. Geulayov G, Casey D, McDonald KC, et al. Incidence of suicide, hospital-presenting non-fatal self-harm, and community-occurring non-fatal self-harm in adolescents in England (the iceberg model of self-harm): a retrospective study. Lancet Psychiatry. 2018;5:167-174. doi:10.1016/S2215 $-0366(17) 30478-9$.

12. Plemmons G, Hall M, Doupnik S, et al. Hospitalization for suicide ideation or attempt: 2008-2015. Pediatrics. 2018;141(6):e20172426. doi:10.1542 /peds.2017-2426. 
13. Spiller HA, Ackerman JP, Smith GA, et al. Suicide attempts by self-poisoning in the United States among 10-25 year olds from 2000 to 2018: substances used, temporal changes and demographics. Clin Toxicol (Phila). 2019:1-12. doi:10.1080/15563650.2019 .1665182

14. Gunnell D, Murray V, Hawton K. Use of paracetamol (acetaminophen) for suicide and nonfatal poisoning: worldwide patterns of use and misuse. Suicide Life Threat Behav. 2000;30(4): 313-326.

15. Santé Canada, Direction des produits thérapeutiques, Direction générale des produits de santé et des aliments. Ligne directrice : Norme d'étiquetage pour l'acétaminophène. Ottawa (Ont.) : Santé Canada; 2009. En ligne à : http://ashleylouisecampbell.com/web _documents/label_stand_guide_ ld-eng.pdf [en anglais seulement]

16. Santé Canada, Direction des produits de santé naturels et sans ordonnance, Direction générale des produits de santé et des aliments. Ligne directrice révisée : Norme d'étiquetage pour l'acétaminophène, Ottawa (Ont.) : Santé Canada; 2016. En ligne à : https://www.hc-sc.gc.ca/dhp-mps /alt_formats/pdf/prodpharma /applic-demande/guide-ld/label_ stand_guide_ld-2016-fra.pdf 